\title{
Pseudo-invariant approach for a particle in a complex time-dependent linear potential
}

\author{
Walid Koussa $^{a *}$, Mustapha Maamache ${ }^{a \dagger}$ \\ ${ }^{(a)}$ Laboratoire de Physique Quantique et Systèmes Dynamiques, \\ Faculté des Sciences, Université Ferhat Abbas Sétif 1, Sétif 19000, Algeria.
}

\begin{abstract}
The Lewis and Riesenfeld method has been investigated, by Ramos et al in Ref. 1], for quantum systems governed by time-dependent $\mathcal{P} \mathcal{T}$ symmetric Hamiltonians and particularly where the quantum system is a particle submitted to action of a complex time-dependent linear potential. We discuss the method they used and propose an alternative one which leads to physically acceptable uncertainty product and to complex $x$ and $p$ expectation values but describe the classical motion. We used, for this situation, a linear pseudo hermitian invariant operator which allow us to solve analytically the time-dependent Schrödinger equation for this problem and to construct a Gaussian wave packet solution. The normalization condition for the invariant eigenfunctions with the Dirac delta function is correctly obtained, contrary to what is stated in Ref. 11.
\end{abstract}

PACS: 03.65.Ca, 03.65.-w

Keywords: Non-Hermitian quantum mechanics, $\mathcal{P} \mathcal{T}$ time-dependent Hamiltonians, $\mathcal{P} \mathcal{T}$ invariant operator, Pseudo-Hermitian invariant operator

\section{Introduction}

One of the "principles" of quantum theory is the association of a Hermitian operator with any physical quantity, a property that guarantees the reality of eigenvalues. In reality, the condition of hermiticity is a sufficient condition, which is by no means necessary, since there are non hermitic operators whose spectrum is real. The central idea is to replace the condition of hermiticity by a weaker condition obviously also ensures the reality of eigenvalues. This led Bender and Boettcher 2 to propose replacing the condition of hermiticity by the parity-time $(\mathcal{P} \mathcal{T}$ ) symmetry, the invariance under simultaneous parity and time reversal transformation, that plays an important role in non-Hermitian quantum mechanics, optics physics, condensed matter and quantum field theory. Starting in quantum mechanics, the concept of $\mathcal{P} \mathcal{T}$ symmetry found applications in many areas of physics [3, 4, 5]. In particular, there is a lot of interest in optics due to experimental

*E-mail: koussawalid@yahoo.com

${ }^{\dagger}$ E-mail: maamache@univ-setif.dz 
realizations of paraxial $\mathcal{P} \mathcal{T}$ symmetric optics [6, 7]. Recent applications include single-mode $\mathcal{P} \mathcal{T}$ lasers [8, 9] and unidirectional reflectionless $\mathcal{P} \mathcal{T}$-symmetric metamaterials at optical frequencies [10]. $\mathcal{P} \mathcal{T}$ symmetric systems demonstrate many nontrivial non-conservative wave interactions and phase transitions, which can be employed for signal ltering and switching, opening new prospects for active control of light [11].

Parity $\mathcal{P}$ has the effect to change the sign of the momentum operator $p$ and the position operator $x$. The anti-linear operator $\mathcal{T}$ has the effect to change the sign of the momentum operator $p$ and the pure imaginary complex number $i$. When an eigenstate of the Hamiltonian is simultaneously an eigenstate of $\mathcal{P} \mathcal{T}$, the eigenvalues are real we call the symmetry unbroken; otherwise the symmetry is broken and the eigenvalues come in complex conjugate pairs and violates the unitarity of the theory. Replacing the standard Hermitian inner product with the obvious choice

$$
\langle\phi \mid \psi\rangle_{\mathcal{P} \mathcal{T}}=\int_{\mathrm{C}} d x[\mathcal{P} \mathcal{T} \phi(x)] \psi(x)
$$

where $\mathcal{P} \mathcal{T} \phi(x)=\phi^{*}(-x)$ and the integral is taken over the contour in the complex- $x$ plane. The advantage of this inner product is that the associated norm $\langle\phi \mid \phi\rangle$ is conserved in time. On unbroken eigenstates $\left|\phi_{n}\right\rangle$ of a $\mathcal{P} \mathcal{T}$-symmetric Hamiltonian, the inner product (1) is (under appropriate assumptions ) pseudo-orthonormal:

$$
\left\langle\phi_{m} \mid \phi_{n}\right\rangle_{\mathcal{P} \mathcal{T}}=(-)^{m} \delta_{m n}
$$

Since the $\mathcal{P} \mathcal{T}$-norm is not positive-definite, to render the energy eigenstates orthonormal is to redefine the inner product (11) by introducing a new symmetry, denoted $\mathcal{C}$ [12, 13, having properties very similar to the charge conjugation operator, inherent in all $\mathcal{P} \mathcal{T}$-symmetric Hamiltonians that possess an unbroken $\mathcal{P} \mathcal{T}$ symmetry. This has allowed to introduce an inner-product structure associated with $\mathcal{C P} \mathcal{T}$ conjugation for which the norms of quantum states are positive definite and unitary-invariant. In particular, $\mathcal{C P} \mathcal{T}$ symmetry is shown to generalize the conventional Hermiticity requirement by replacing it with a dynamically determined inner product (one that is defined by the Hamiltonian itself). Several authors have studied time independent quantum systems governed by non-Hermitian Hamiltonians [14, 15, 16, 17, 18, 19, 20, 21.

Even before the discovery of $\mathcal{P} \mathcal{T}$-symmetry and the introduction of the $\mathcal{C P} \mathcal{T}$-inner product, there have been very general considerations [22] addressing the question of how a consistent quantum mechanical framework can be constructed from the non-Hermitian Hamiltonian systems. It was understood at that time that quasi-Hermitian systems [22] would lead to positive inner products. It has been clarified [23, 24, 25, 26] that a non-Hermitian Hamiltonian having all eigenvalues real is connected to its Hermitian conjugate,

$$
H^{\dagger}=\eta H \eta^{-1}
$$

through a linear, Hermitian, invertible and bounded metric operator $\eta=\rho^{+} \rho$ with a bounded inverse, i.e. $H$ is Hermitian with respect to a positive definite inner product $\langle., .\rangle_{\eta}=\langle.|\eta|$. defined as

$$
\left\langle\phi_{m}^{H} \mid \phi_{n}^{H}\right\rangle_{\eta}=\left\langle\phi_{m}^{H}|\eta| \phi_{n}^{H}\right\rangle=\delta_{m n}
$$

and called $\eta$-pseudo-Hermitian inner product. It is also established [23, 24, 25, 26] that the non Hermitian Hamiltonian (or a pseudo-Hermitian Hamiltonian) $H$ can be transformed to an 
equivalent Hermitian one given by

$$
h=\rho H \rho^{-1}
$$

where $h$ is the equivalent Hermitian analog of $H$ with respect to the standard inner product $\langle.,.\rangle \cdot \rho$ is often called the Dyson map [27]. Thus, although the eigenvalue spectra of $h$ and $H$ are identical, relations between their eigenvectors will differ

$$
\left|\psi_{n}^{h}\right\rangle=\rho\left|\phi_{n}^{H}\right\rangle
$$

All these efforts have been devoted to study time-independent non-Hermitian systems. Whereas the treatment for systems with time-dependent non-Hermitian Hamiltonians with time-independent or time-dependent an metric operators have been extensively studied [28, 29, 30, 31, 32, 33, 34, 35, 36, 37, 38, 39, 40, 41, 42, 43, 44, 45, 46, 47, 48, 49, 50, 51, 52, 53, 54, 55, 56, 57, 58, 59, 60. Nevertheless, the existence of invariants (constants of the motion or first integral) introduced by Lewis- Riesenfeld [61] is a factor of central importance in the study of time-dependent systems.

While research on $\mathcal{P} \mathcal{T}$-symmetry has focused on time-independent Hamiltonians, very few works using a $\mathcal{P} \mathcal{T}$-symmetric time-dependent Hamiltonians, where the time-reversal operator $\mathcal{T}$ has also the effect to change the sign of the time $t \rightarrow-t$ and whose action on the wave function defined as [62, 63, 64]

$$
\mathcal{T} \psi(x, t)=\psi^{*}(x,-t)
$$

is barely found in the literature [65, 66, 67, 68, 69, 70, 71].

In a recent paper, Ramos et al [1] extend the well-known Lewis and Riesenfeld invariant method [61] to $\mathcal{P} \mathcal{T}$-symmetric time-dependent non-Hermitian Hamiltonians and apply it to the quantum motion of a particle in the presence of a complex time-dependent linear potential with $\mathcal{P} \mathcal{T}$ - symmetry. They have misleadingly claim that the invariant eigenstates normalization condition associated with the Dirac delta function is verified.

The main objective in this paper is to give, in section 2 , a brief recall of results discussed by Ramos et al [1] on the motion of a particle under the action of a complex time dependent $\mathcal{P} \mathcal{T}$ -symmetric linear potential. After that, we discuss the misleadingly results concerning the $\mathcal{P} \mathcal{T}$ inner product of the simultaneously eigenstates of the $\mathcal{P} \mathcal{T}$ operator and the $\mathcal{P} \mathcal{T}$-symmetric invariant operator $I^{\mathcal{P} \mathcal{T}}(t)$. In section 3 , we give an alternative method based on the pseudoHermitian invariant operator [44, 54] to find the solutions for a particle submitted to the action of a complex time-dependent linear potential. Finally in section 4, we construct the Gaussian wave packet state for this problem. Despite that the expectation values of the $x$ and $p$ operators are complex, they are identical to the classical variables $x_{c}, p_{c}$. In addition, we obtain that the uncertainty product is physically acceptable.

\section{$2 \mathcal{P} \mathcal{T}$-symmetric invariant operator $I^{\mathcal{P}} \mathcal{T}(t)$ for complex time- dependent linear potential}

B.F.Ramos et al [1] have investigated the motion quantum of a particle with time-dependent mass subject to the action of a complex time-dependent linear potential described as

$$
H(t)=\frac{p^{2}}{2 m(t)}+i f(t) x
$$


where $f(t)$ is a real time-dependent function. The classical variables describing the equations of motion are given by

$$
\dot{p}_{c}=-\frac{\partial H}{\partial x_{c}}=-i f(t)
$$

and

$$
\dot{x}_{c}=\frac{\partial H}{\partial p_{c}}=\frac{p_{c}}{m(t)}
$$

By solving the above two equations, the space and momentum operators can be obtained in terms of the initial conditions, given by

$$
p_{c}=p_{0}-i \int_{0}^{t} f\left(t^{\prime}\right) d t^{\prime}
$$

and

$$
x_{c}=x_{0}+p_{0} \int_{0}^{t} \frac{d t^{\prime}}{m\left(t^{\prime}\right)}-i\left(\int_{0}^{t} \frac{d t^{\prime}}{m\left(t^{\prime}\right)} \int_{0}^{t^{\prime}} f(\tau) d \tau\right)
$$

By extending the well-known Lewis and Riesenfeld invariant method, they looked for a $\mathcal{P} \mathcal{T}$ symmetric non-Hermitian time-dependent linear operator given by

$$
I(t)=a(t) x+b(t) p+c(t)
$$

where $a(t), b(t)$ and $c(t)$ are complex time-dependant c-number functions.

Inserting the invariant $I(t)$ in the Van-Neumann equation

$$
i \frac{\partial I(t)}{\partial t}=[H(t), I(t)]
$$

and after some algebra, gives

$$
\begin{aligned}
& a(t)=a_{0} \\
& b(t)=-a_{0} \int_{0}^{t} \frac{d \tau}{m(\tau)} \\
& c(t)=-i a_{0} \int_{0}^{t} d \tau f(\tau) \int_{0}^{\tau} \frac{d \tau^{\prime}}{m\left(\tau^{\prime}\right)}
\end{aligned}
$$

on the other hand, the $\mathcal{P} \mathcal{T}$ symmetric invariant operator condition

$$
I^{\mathcal{P} \mathcal{T}}(t)=(\mathcal{P} \mathcal{T}) I(t)(\mathcal{P} \mathcal{T})=I(t)
$$

provides

$$
\begin{aligned}
a_{0} & =-a_{0}^{*} \\
b^{*}(t) & =-b(t) \\
c^{*}(t) & =c(t)
\end{aligned}
$$


Using the transformation

$$
\varphi_{\lambda}(x, t) \rightarrow \phi_{\lambda}(x, t)=e^{-i \frac{\theta_{\lambda}}{2}} \varphi_{\lambda}(x, t)
$$

and solving the eigenequation

$$
I^{\mathcal{P} \mathcal{T}}(t) \phi_{\lambda}(x, t)=\lambda \phi_{\lambda}(x, t)
$$

so that the eigenfunctions (Eq. (37) of Ref. [1]) are given by

$$
\begin{aligned}
\phi_{\lambda}(x, t) & =\sqrt{\frac{\sigma_{\lambda}}{2 \pi \hbar b(t)}} \exp \left\{\frac{i}{\hbar b(t)}\left[(\lambda-c(t)) x-\frac{a_{0}}{2} x^{2}\right]\right\}, \\
\sigma_{\lambda} & = \pm 1
\end{aligned}
$$

Without loss of generalities, we drop the phase factor $e^{-i \frac{\theta_{\lambda}}{2}}$. Thus, the eigenstates $\phi_{\lambda}(x, t)$ of $I^{\mathcal{P} \mathcal{T}}(t)$ are eigenstates of the $\mathcal{P} \mathcal{T}$ operator with eigenvalue 1 , when the action of $\mathcal{P} \mathcal{T}$ operator on the wave function is as follows

$$
\begin{aligned}
\mathcal{P} \mathcal{T} \phi_{\lambda}(x, t) & =\phi_{\lambda}^{*}(-x,-t) \\
& =\sqrt{\frac{\sigma_{\lambda}}{2 \pi b^{*}(-t)}} \exp \left\{\frac{-i}{b^{*}(-t)}\left[\left(\lambda-c^{*}(-t)\right)(-x)-\frac{a_{0}^{*}}{2} x^{2}\right]\right\} \\
& =\sqrt{\frac{\sigma_{\lambda}}{2 \pi b(t)}} \exp \left\{\frac{i}{b(t)}\left[(\lambda-c(t)) x-\frac{a_{0}}{2} x^{2}\right]\right\}=\phi_{\lambda}(x, t) .
\end{aligned}
$$

On the other hand, the authors of Ref.[1] claim, incorrectly, that the normalization condition (Eq. (39) in [1]) associated with the Dirac delta function is verified. To see that their assertion is not correct, it is enough to calculate explicitly the $\mathcal{P} \mathcal{T}$ inner product defined as

$$
\begin{aligned}
\int_{-\infty}^{+\infty}\left[\phi_{\lambda^{\prime}}(x, t)\right]^{\mathcal{P T}} \phi_{\lambda}(x, t) d x & =\int_{-\infty}^{+\infty} \phi_{\lambda^{\prime}}^{*}(-x,-t) \phi_{\lambda}(x, t) d x=\int_{-\infty}^{+\infty} \phi_{\lambda^{\prime}}(x, t) \phi_{\lambda}(x, t) d x \\
& =\frac{\sqrt{\sigma_{\lambda^{\prime}} \sigma_{\lambda}}}{2 \pi b(t)} \int_{-\infty}^{+\infty} \exp \left\{\frac{i}{b(t)}\left[\left(\left(\lambda+\lambda^{\prime}\right)-2 c(t)\right) x-a_{0} x^{2}\right]\right\} d x \neq \delta\left(\lambda-\lambda^{\prime}\right)
\end{aligned}
$$

According to the invariant operator theory, the time-dependent Schrödinger equation takes the form

$$
\psi_{\lambda}(x, t)=e^{i \mu_{\lambda}(t)} \phi_{\lambda}(x, t),
$$

where the phase functions are given by

$$
\mu_{\lambda}(t)=-\frac{1}{2 \hbar} \int_{0}^{t} \frac{(\lambda-c(\tau))^{2}}{m(\tau) b^{2}(\tau)} d \tau .
$$

So, they construct a Gaussian wave packet solution

$$
\Psi(x, t)=\int_{-\infty}^{+\infty} g(\lambda) \psi_{\lambda}(x, t) d \lambda
$$


where the Gaussian weight function $g(\lambda)$ is given by

$$
g(\lambda)=\frac{\sqrt{d}}{\sqrt{2 \pi}} \exp \left[-\frac{d^{2}}{4} \lambda^{2}\right] .
$$

Thus, the general solution (25) is given by equation (47) of Ref.[1]. Using this wave packet solution, they calculate the expectation values of the position $\langle x\rangle_{\mathcal{P} \mathcal{T}}$ and the momentum $\langle p\rangle_{\mathcal{P} \mathcal{T}}$ as well as the uncertainty product $\Delta x . \Delta p$, where the expectation value $\langle O\rangle_{\mathcal{P} \mathcal{T}}$ of an operator $O$ is defined as

$$
\int_{-\infty}^{\infty} d x \Psi(x, t)^{\mathcal{P} \mathcal{T}} O \Psi(x, t)
$$

They state that the $\mathcal{P} \mathcal{T}$ operator acts on the wave function as follows [72]

$$
\mathcal{P} \mathcal{T} \Psi(x, t)=\Psi(x, t)^{\mathcal{P} \mathcal{T}}=\Psi^{*}(-x, t),
$$

which is in contrast with the definition (21) employed to show that the eigenstates of the linear invariant are also eigenstates of the $\mathcal{P} \mathcal{T}$ operator.

However, knowing that the wave function is a scalar and taking into account that $\mathcal{T}$ is antilinear and antiunitary operator, we obtain the time reversal rule for the wave function $\mathcal{T} \Psi(x, t)=\Psi^{*}(x,-t)$. That is the general rule for time reversal in quantum mechanics: if a certain state is described by the wave function $\Psi(x, t)$, then the "time-reversed" state is described by the function $\Psi^{*}(x,-t)$. The change to the complex conjugate function is necessary because the "correct" time dependence must be restored, after being lost through the change in the sign of $t$ 62.

Finally, they find that the expectation values $\langle x\rangle_{\mathcal{P} \mathcal{T}},\langle p\rangle_{\mathcal{P} \mathcal{T}}$ are imaginary numbers so that the position, momentum operators are not observables. As a consequence, the uncertainty relation $\Delta x . \Delta p$, which is a complex number, is physically unacceptable.

In the next section, using the pseudo-Hermitian invariant operator approach [44, 54 we get an accepted physical quantities for a " Particle in a complex time-dependent linear potential ". We show that the expectation values of $x$ and $p$ are complex numbers that describe the classical motion while the uncertainty relation is physically acceptable. On the other hand, the normalization condition for the invariant eigenfunctions with the Dirac delta function is verified.

\section{The complex time-dependent linear potential:pseudo-invariant method}

The beginning of this section briefly recalls the results of the pseudo-invariant operator technique [44, 54]. In complete analogy to the time independent scenario a self-adjoint invariant operator $I^{h}(t)$, i.e., an observable, in the Hermitian system which has an observable counterpart $I^{P H}(t)$ in the non-Hermitain system are related to each other as $I^{h}(t)=\rho(t) I^{P H}(t) \rho^{-1}(t) \Leftrightarrow I^{P H \dagger}(t)=$ $\eta(t) I^{P H}(t) \eta^{-1}(t)$ was introduced and adressed in details in Ref. [44, 54] that we will briefly recall. Given a non-Hermitian time-dependent Hamiltonian operator $H(t)$, it is possible to build a pseudo-invariant operator $I^{P H}(t)$ verifying

$$
\frac{d I^{P H}(t)}{d t}=\frac{\partial I^{P H}(t)}{\partial t}-i\left[I^{P H}(t), H(t)\right]=0
$$


and obeys the eigenvalue equation:

$$
I^{P H}(t)\left|\phi_{n}^{H}(t)\right\rangle=\lambda_{n}\left|\phi_{n}^{H}(t)\right\rangle,
$$

where the eigenvalues $\lambda_{n}$ are time-independent and the eigenstates $\left|\phi_{n}^{H}(t)\right\rangle$ of $I^{P H}(t)$ are orthonormal

$$
\left\langle\phi_{m}^{H}(t)|\eta(t)| \phi_{n}^{H}(t)\right\rangle=\delta_{m, n} .
$$

The solutions of the Schrödinger equation

$$
i \hbar \frac{\partial}{\partial t}\left|\Phi^{H}(t)\right\rangle=H(t)\left|\Phi^{H}(t)\right\rangle
$$

can be written in terms of the eigenfunctions $\left|\phi_{n}^{H}(t)\right\rangle$ as

$$
\left|\Phi_{n}^{H}(t)\right\rangle=e^{i \varphi_{n}(t)}\left|\phi_{n}^{H}(t)\right\rangle
$$

where the phase functions $\varphi_{n}(t)$ are found from the equation:

$$
\frac{d \varphi_{n}(t)}{d t}=\left\langle\phi_{n}^{H}(t)\left|\eta(t)\left[i \frac{\partial}{\partial t}-\frac{H(t)}{\hbar}\right]\right| \phi_{n}^{H}(t)\right\rangle .
$$

For a particle with time-dependent mass subject to the action of a complex time-dependent linear potential described by the Hamilonian (86), we choose a linear pseudo-Hermitian invariant operator $I^{P H}(t)$ in the form

$$
I^{P H}(t)=a(t)\left(x-\frac{i}{2} \alpha(t)\right)+b(t)\left(p-\frac{i}{2} \beta(t)\right)+c(t)
$$

where $\alpha(t)$ and $\beta(t)$ are real parameters while $a(t), b(t)$ and $c(t)$ are time-dependent $c$-number functions to be determined.

The condition (28) implies that

$$
\begin{aligned}
\dot{a}(t) & =0 \\
\dot{b}(t) & =-\frac{a}{m(t)} \\
\dot{c}(t)-\frac{i}{2}(\dot{\alpha} a+\dot{\beta} b+\beta \dot{b}) & =i b f(t)
\end{aligned}
$$

after solving these equations, we get

$$
\begin{aligned}
a(t) & =a_{0} \\
b(t) & =b_{0}-a_{0} \int_{0}^{t} \frac{1}{m\left(t^{\prime}\right)} d t^{\prime} \\
c(t) & =c_{0} \\
f(t) & =-\frac{1}{2 b}\left(\dot{\alpha} a_{0}+\dot{\beta} b-\beta \frac{a_{0}}{m(t)}\right)
\end{aligned}
$$


Since the operator $I^{P H}(t)$ is pseudo-Hermitian, then it fulfills the condition

$$
I^{+P H}(t)=\eta(t) I^{P H}(t) \eta^{-1}(t)
$$

where the operator metric $\eta(t)$ is chosen as

$$
\eta=\rho^{+} \rho=\exp [\beta(t) x-\alpha(t) p] .
$$

The condition (37) provides

$$
\begin{aligned}
a(t) & =a^{*}(t) \\
b(t) & =b^{*}(t) \\
c(t) & =c^{*}(t)
\end{aligned}
$$

To find a solution of the Schrödinger equation of $H(t)$

$$
H(t) \Psi^{H}(x, t)=i \partial_{t} \Psi^{H}(x, t),
$$

where $\Psi^{H}(x, t)=\int_{-\infty}^{+\infty} g(\lambda) \psi_{\lambda}(x, t) d \lambda$ and $\psi_{\lambda}(x, t)=e^{i \mu_{\lambda}(t)} \varphi_{\lambda}^{I^{P H}}(x, t) ; \mu_{\lambda}(t)$ should be real, we have first to solve the eigenvalue equation of the invariant $I^{P H}(t) \varphi_{\lambda}^{I^{P H}}(x, t)=\lambda \varphi_{\lambda}^{I^{P H}}(x, t)$. After some basic calculations, we get that the orthonormalized solutions of the invariant eigenvalue equation are given by

$$
\varphi_{\lambda}^{I^{P H}}(x, t)=\frac{1}{\sqrt{2 \pi b}} \exp \frac{i}{2 b}\left[(2(\lambda-c)+i \beta b)\left(x-\frac{i}{2} \alpha\right)-a_{0}\left(x-\frac{i}{2} \alpha\right)^{2}\right]
$$

by subtituting $\varphi_{\lambda}^{I^{P H}}(x, t)$ (41) multiplied by a phase factor $e^{i \mu_{\lambda}(t)}$ in the Schrödinger equation $H(t) \psi_{\lambda}(x, t)=i \partial_{t} \psi_{\lambda}(x, t)$, we get

$$
\begin{aligned}
\dot{\mu}_{\lambda} \varphi_{\lambda}^{I^{P H}}(x, t) & =\left[-\frac{1}{2 m(t) b^{2}}(\lambda-c)^{2}+\frac{1}{2}\left(\alpha f-\frac{\dot{\alpha}}{2} \beta+\frac{\beta^{2}}{4 m(t)}\right)\right. \\
& \left.-\frac{i}{2 b}\left(\frac{\beta}{m(t)}-\dot{\alpha}\right)(\lambda-c)\right] \varphi_{\lambda}^{I^{P H}}(x, t),
\end{aligned}
$$

since this phase should be real, it implies that $m \dot{\alpha}=\beta$, this is equivalent to $f(t)=-\dot{\beta}(t) / 2$. The phase equation (42) is simplified into

$$
\dot{\mu}_{\lambda}=\left[-\frac{1}{2 m(t) b^{2}}(\lambda-c)^{2}-\frac{1}{4}\left(\dot{\beta} \alpha+\frac{\beta^{2}}{2 m(t)}\right)\right] .
$$

So that the general solution can written as

$$
\Psi^{H}(x, t)=\int_{-\infty}^{+\infty} g(\lambda) e^{i \mu_{\lambda}} \varphi_{\lambda}^{I^{P H}}(x, t) d \lambda
$$

we choose the weight function $g(\lambda)$ in the form

$$
g(\lambda)=\sqrt{\frac{\sqrt{d}}{\pi \sqrt{2 \pi} b_{0}}} \exp \left[-d\left(\lambda-I_{0}\right)^{2}\right] \exp \left[-i \frac{d_{0}}{b_{0}}\left(\lambda-\frac{I_{0}}{2}\right)\right]
$$


where $d, d_{0}, I_{0}$ are positive real constants.

After a straighforward calculation, we obtain the general expression solution in form of the Gaussian wave-packet

$$
\begin{aligned}
\Psi^{H}(x, t) & =\sqrt{\frac{\sqrt{d}}{\sqrt{2 \pi} b\left(i \int \frac{1}{2 m b^{2}}+d\right)}} \exp \left\{-i \int_{0}^{t} \frac{\left(c-I_{0}\right)^{2}}{2 m b^{2}} d t^{\prime}\right\} \\
& \exp \left\{-i \frac{I_{0}}{2} \frac{d_{0}}{b_{0}}\right\} \exp \left\{-\frac{i}{4} \int_{0}^{t}\left(\dot{\beta} \alpha+\frac{\beta^{2}}{2 m}\right) d t^{\prime}\right\} \\
& \exp \left\{\frac{i}{2 b}\left[\left(-2\left(c-I_{0}\right)+i \beta b\right)\left(x-\frac{i}{2} \alpha\right)-a_{0}\left(x-\frac{i}{2} \alpha\right)^{2}\right]\right\} \\
& \exp \left\{-\frac{\left[\left(x-\frac{i}{2} \alpha\right)-b\left(-\int_{0}^{t} \frac{\left(c-I_{0}\right)}{m b^{2}} d t^{\prime}+\frac{d_{0}}{b_{0}}\right)\right]^{2}}{4 b^{2}\left(i \int_{0}^{t} \frac{1}{2 m b^{2}} d t^{\prime}+d\right)}\right\}
\end{aligned}
$$

Now, we calculate the expectation values of the position and momentum operators in the Gaussian state $\Psi^{H}(x, t)$

$$
\begin{aligned}
& \langle x\rangle_{\eta}=\left\langle\Psi^{H}(t)|\eta x| \Psi^{H}(t)\right\rangle=d_{0}-\frac{c_{0}}{b_{0}} \int_{0}^{t} \frac{d t^{\prime}}{m\left(t^{\prime}\right)}+\frac{i}{2} \alpha \\
& \langle p\rangle_{\eta}=\left\langle\Psi^{H}(t)|\eta p| \Psi^{H}(t)\right\rangle=-\frac{c_{0}}{b_{0}}+\frac{i}{2} \beta
\end{aligned}
$$

it is obvious that $\langle x\rangle_{\eta}$ and $\langle p\rangle_{\eta}$ are identical to the classical variables $x_{c}, p_{c}$

$$
\langle x\rangle_{\eta}=x_{c} \quad, \quad\langle p\rangle_{\eta}=p_{c}
$$

We also evaluate the uncertainty in the position and the momentum

$$
\begin{gathered}
\Delta x=\sqrt{\left\langle x^{2}\right\rangle_{\eta}-\left(\langle x\rangle_{\eta}\right)^{2}}=\frac{b}{\sqrt{d}} \sqrt{d^{2}+\left(\int_{0}^{t} \frac{d t^{\prime}}{2 m\left(t^{\prime}\right) b^{2}}\right)^{2}} \\
\Delta p=\sqrt{\left\langle p^{2}\right\rangle_{\eta}-\left(\langle p\rangle_{\eta}\right)^{2}}=\frac{1}{\Delta x} \sqrt{\frac{1}{4}+\left[\frac{1}{4 b_{0} d b} \int_{0}^{t} \frac{d t^{\prime}}{m\left(t^{\prime}\right)}-\frac{a_{0}}{b}(\Delta x)^{2}\right]^{2}}
\end{gathered}
$$

as well as the uncertainty product

$$
\Delta p \Delta x=\sqrt{\frac{1}{4}+\left[\frac{1}{4 b_{0} d b} \int_{0}^{t} \frac{d t^{\prime}}{m\left(t^{\prime}\right)}-\frac{a_{0}}{b}(\Delta x)^{2}\right]^{2}} \geqslant \frac{1}{2},
$$

which is real and greater than (or equal ) to $1 / 2$ and therefore physically acceptable. 
Figure1.a.

Variances of the physical position $(\Delta x)^{2}$

(Dashed-blue) and momentum $(\Delta p)^{2}$ (solid-red), with the following

parameters: $\left(q_{0}=p_{0}=a_{0}=m=d=\right.$

$1, b_{0}=2$ and $\left.\hbar=1\right)$.

Figure1.b.

The uncertainty product with the same parameters as in figure (a). 
The density $\left|\rho \Psi^{H}(x, t)\right|^{2}$ can be written in function of $\langle x\rangle_{\eta}$ and $\Delta x$ as

$$
\left|\rho \Psi^{H}(x, t)\right|^{2}=\left|\sqrt{\frac{\sqrt{d}}{\sqrt{2 \pi} b\left(i \int_{0}^{t} \frac{d t^{\prime}}{2 m b^{2}}+d\right)}}\right|^{2} \exp -\frac{\left[x-\left(x_{c}-\frac{i}{2} \alpha\right)\right]^{2}}{2 \Delta x^{2}}
$$

and represents a Gaussian with center in $\left(\langle x\rangle_{\eta}-\frac{i}{2} \alpha\right)=\left(x_{0}+p_{0} \int \frac{1}{m}\right)$ and time-dependent width $\Delta x$ and therefore

$$
\int_{-\infty}^{+\infty}\left|\rho \Psi^{H}(x, t)\right|^{2} d x=1
$$

Figure 2. Probability density $\left|\rho \Psi^{H}(x, t)\right|^{2}$ for Gaussian wave packet with $q_{0}=p_{0}=a_{0}=m=d=1, b_{0}=2$ and $\hbar=1$. The horizontal and vertical axes correspond to position and time, respectively.

In summary, using the time-dependent pseudo-Hermitian linear invariant method, we have found the solutions for a particle submitted to the action of a complex time-dependent linear potential. Furthermore, we have constructed a Gaussian wave packet state for our problem and shown that the time-dependent probability density associated with this packet is Gaussian and remains Gaussian for all time. In addition, the expected values of the operators $x$ and $p$, even though that are complex numbers, represent the classical solutions. We have found that the uncertainty product is physically acceptable. Also, the normalization condition for the invariant eigenfunctions with the Dirac delta function is correctly obtained. 


\section{References}

[1] B.F. Ramos, I.A. Pedrosa, and Alberes Lopes de Lima, "Lewis and Riesenfeld approach to time-dependent non-Hermitian Hamiltonians having $\mathcal{P} \mathcal{T}$ symmetry,"

Eur. Phys. J. Plus 133, 449 (2018).

[2] Carl M. Bender and Stefan Boettcher, "Real spectra in non-Hermitian Hamiltonians having $\mathcal{P} \mathcal{T}$ symmetry," Phys. Rev. Lett. 80, 5243-5246 (1998).

[3] Carl M. Bender, B. Berntson, D. Parker and E. Samuel, "Observation of $\mathcal{P} \mathcal{T}$ phasetransition in a simple mechanical system," Am. J. Phys. 81, 173\{179 (2013).

[4] J. Rubinstein, P. Sternberg and Q. Ma, "Bifurcation diagram and pattern formation of phase slip centers in superconducting wires driven with electric currents," Phys. Rev. Lett. 99, 167003 (2007).

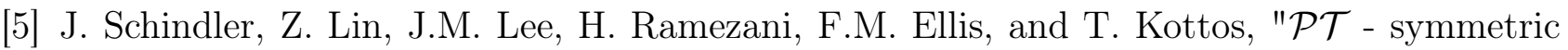
electronics," J. Phys. A. 45, 444029 (2012).

[6] K.G. Makris, R. El-Ganainy, D.N. Christodoulides, and Z.H. Musslimani, "Beam Dynamics in $\mathcal{P} \mathcal{T}$ Symmetric Optical Lattices," Phys. Rev. Lett. 100, 103904 (2008).

[7] Z.H. Musslimani, K.G. Makris, R. El-Ganainy, and D.N. Christodoulides, "Optical Solitons in $\mathcal{P} \mathcal{T}$ Periodic Potentials," Phys. Rev. Lett. 100, 030402 (2008).

[8] L. Feng, Z.J. Wong, R. Ma, Y. Wang, and X. Zhang, "Single-mode laser by parity time symmetry breaking," Science 346, 972 (2014).

[9] H. Hodaei, M.-A. Miri, M. Heinrich, D.N. Christodoulides, and M. Khajavikhan, "Paritytime-symmetric microring lasers," Science 346, 975 (2014).

[10] L. Feng, Y.-L. Xu, W.G. Fegadolli, M.-H. Lu, J.E.B. Oliveira, V.R. Almeida, Y.-F. Chen, and A. Scherer, Experimental demonstration of a unidirectional reflectionless parity-time metamaterial at optical frequencies," Nat. Matter 12, 108 (2013).

[11] S.V. Suchkov, A.A. Sukhorukov, J. Huang, S.V. Dmitriev, C. Lee, Yu.S. Kivshar, "Nonlinear switching and solitons in $\mathcal{P} \mathcal{T}$-symmetric photonic systems," Laser Photonics Rev. 10, 177 (2016).

[12] Carl M. Bender, Dorje C. Brody, and Hugh F. Jones, "Complex Extension of Quantum Mechanics," Phys. Rev. Lett. 89, 270401 (2002).

[13] Carl M. Bender, "Making sense of non-Hermitian Hamiltonians," Rep. Prog. Phys. 70, 947 (2007).

[14] B. Bagchi, C. Quesne, M. Znojil, "Generalized Continuity Equation and Modified Normalization in $\mathcal{P} \mathcal{T}$-Symmetric Quantum Mechanics," Mod. Phys. Lett. A 16, 2047 (2001).

[15] Z. Ahmed, "Real and complex discrete eigenvalues in an exactly solvable one-dimensional complex $\mathcal{P} \mathcal{T}$-invariant potential," Phys. Lett. A 282, 343 (2001). 
[16] M. Znojil, "Solvable simulation of a double-well problem in PT -symmetric quantum mechanics," J. Phys. A 36,7639 (2003).

[17] S. Weigert, "Completeness and orthonormality in $\mathcal{P} \mathcal{T}$-symmetric quantum systems," Phys. Rev. A 68, 062111 (2003).

[18] Z. Ahmed, "P-, $\mathcal{T}$-, $\mathcal{P} \mathcal{T}_{-}$, and $\mathcal{C} \mathcal{P} \mathcal{T}$-invariance of Hermitian Hamiltonians," Phys. Lett. A 310, 139 (2003).

[19] S. Weigert, "Detecting broken $\mathcal{P} \mathcal{T}$-symmetry," J. Phys. A.39, 10239 (2006).

[20] Z. Ahmed, "Eigenvalue problems for the complex $\mathcal{P} \mathcal{T}$-symmetric potential $V(x)=$ igx," Phys. Lett. A 364, 12 (2007).

[21] J. da Providência, N. Bebiano, J. P. da Providência, "Non-Hermitian Hamiltonians with Real Spectrum in Quantum Mechanics," Braz. J. Phys. 41, 78 (2011).

[22] F. G. Scholtz, H. B. Geyer, and F. J.W. Hahne, "Quasi-Hermitian operators in quantum mechanics and the variational principle," Ann. Phys. 213, 74-101 (1992).

[23] A. Mostafazadeh, "Pseudo-Hermiticity versus $\mathcal{P} \mathcal{T}$ symmetry: The necessary condition for the reality of the spectrum of a non-Hermitian Hamiltonian," J. Math. Phys. 43, 205 (2002).

[24] A. Mostafazadeh, " Pseudo-Hermiticity versus $\mathcal{P} \mathcal{T}$-symmetry. II. A complete characterization of non-Hermitian Hamiltonians with a real spectrum," J. Math. Phys. 43, 2814 (2002)..

[25] A. Mostafazadeh, "Pseudo-Hermiticity versus $\mathcal{P} \mathcal{T}$-symmetry III: Equivalence of pseudoHermiticity and the presence of antilinear symmetries," J. Math. Phys. 43, 3944 (2002).

[26] A. Mostafazadeh, "Pseudo-Hermitian representation of quantum mechanics," Int. J. Geom. Methods Mod. Phys. 07, 1191 (2010).

[27] F.J. Dyson, " Thermodynamic behavior of an ideal ferromagnet," Phys. Rev. 102, 1230 (1956).

[28] C. Figueira de Morisson Faria and A. Fring, "Time evolution of non-Hermitian Hamiltonian systems," J. Phys. A: Math. Theor. 39, 9269 (2006).

[29] C. Figueira de Morisson Faria and A. Fring, "Non-Hermitian Hamiltonians with real eigenvalues coupled to electric fields: From the time-independent to the time-dependent quantum mechanical formulation," Laser Physics 17, 424 (2007).

[30] A.Mostafazadeh, "Time-dependent pseudo-Hermitian Hamiltonians defining a unitary quantum system and uniqueness of the metric operator," Phys. Lett. B 650, 208 (2007).

[31] M. Znojil, "Time-dependent version of crypto-Hermitian quantum theory," Phys. Rev. D 78, 085003 (2008).

[32] M. Znojil, "Three-Hilbert-space formulation of quantum mechanics," SIGMA 5. 001 (2009). 
[33] H. B'1la, "Adiabatic time-dependent metrics in PT-symmetric quantum theories", eprint arXiv: 0902.0474 (2009).

[34] J. Gong and Q. H. Wang, "Geometric phase in PT -symmetric quantum mechanics," Phys. Rev. A 82, 012103 (2010).

[35] J. Gong and Q. H. Wang, "Timedependent PT -symmetric quantum mechanics," J. Phys. A 46, $485302(2013)$.

[36] M. Znojil, "Crypto-unitary forms of quantum evolution operators," Int. J. Theor. Phys. 52, 2038 (2013).

[37] M. Maamache, "Periodic pseudo-Hermitian Hamiltonian: Nonadiabatic geometric phase," Phys. Rev. A 92, 032106 (2015).

[38] M. Znojil, "Non-Hermitian Heisenberg representation," Phys. Lett. A 379, 2013 (2015).

[39] A. Fring and M. H. Y. Moussa, "Unitary quantum evolution for time-dependent quasiHermitian systems with nonobservable Hamiltonians," Phys. Rev. A 93, 042114 (2016).

[40] A. Fring and M.H. Y. Moussa, "Non-Hermitian Swanson model with a time-dependent metric," Phys. Rev. A 94, 042128 (2016).

[41] Yan-Gang Miao and Zhen-Ming Xu, "Investigation of non-Hermitian Hamiltonians in the Heisenberg picture," Phys. Lett. A 380, 1805 (2016).

[42] F. S. Luiz, M. A. Pontes and M. H. Y. Moussa, "Unitarity of the time-evolution and observability of non-Hermitian Hamiltonians for time-dependent Dyson maps," arXiv:1611.08286 (2016).

[43] B. Khantoul, A. Bounames and M. Maamache, "On the invariant method for the timedependent non-Hermitian Hamiltonians, " Eur. Phys. J. Plus 132: 258 (2017).

[44] M. Maamache, O-K. Djeghiour, N. Mana and W. Koussa, "Pseudo-invariants theory and real phases for systems with non-Hermitian time-dependent Hamiltonians, " Eur. Phys. J. Plus 132, 383 (2017).

[45] A. Fring and T. Frith, "Exact analytical solutions for timedependent Hermitian Hamiltonian systems from static unobservable non-Hermitian Hamiltonians," Phys. Rev. A 95, 010102(R) (2017).

[46] F. S. Luiz, M. A. de Pontes and M. H. Y. Moussa, "Gauge linked time-dependent nonHermitian Hamiltonians," arXiv:1703.01451 (2017).

[47] M. Maamache, "Non-unitary transformation of quantum time-dependent non-Hermitian systems," Acta Polytech. 57, 424 (2017).

[48] M. Znojil, "Non-Hermitian interaction representation and its use in relativistic quantum mechanics," Annals of Physics 385, 162 (2017). 
[49] A. Fring and T. Frith, "Mending the broken PT -regime via an explicit time-dependent Dyson map," Phys. Lett. A 381, 2318 (2017).

[50] A. Fring and T. Frith, "Metric versus observable operator representation, higher spin models," Eur. Phys. J. 133, 57 (2018).

[51] A. Fring and T. Frith, "Time-dependent metric for the two-dimensional, non-Hermitian coupled oscillator," arXiv:1812.02862 (2018).

[52] A. Fring and T. Frith, "Solvable two-dimensional time dependent non-Hermitian quantum systems with infinite dimensional Hilbert space in the broken PT -regime," J. Phys. A.51, 265301 (2018).

[53] Jiangbin Gong and Qing-hai Wang, "Piecewise adiabatic following in non-Hermitian cycling" Phys. Rev. A. 97, 052126 (2018).

[54] W. Koussa, N. Mana, O- K. Djeghiour, and M. Maamache, "The pseudo Hermitian invariant operator and time-dependent non-Hermitian Hamiltonian exhibiting a $\mathrm{SU}(1,1)$ and $\mathrm{SU}(2)$ dynamical symmetry" J. Math. Phys. 59, 072103 (2018).

[55] B. Bagchi, "Evolution operator for time-dependent non-Hermitian Hamiltonians," Lett. High. Energy. Physics. 3, 04 (2018).

[56] A. Fring and T. Frith, "Quasi-exactly solvable quantum systems with explicitly timedependent Hamiltonians," Phys. Lett. A 383, 158 (2019).

[57] Jiangbin Gong and Qing-hai Wang, "Piecewise adiabatic following: General analysis and exactly solvable models" Phys. Rev. A. 99, 012107 (2019).

[58] Da-Jian Zhang, Qing-hai Wang, and Jiangbin Gong, "Quantum geometric tensor in $\mathcal{P} \mathcal{T}$ symmetric quantum mechanics" Phys. Rev. A 99, 042104 (2019).

[59] Da-Jian Zhang, Qing-hai Wang, and Jiangbin Gong, "Time-dependent $\mathcal{P} \mathcal{T}$-symmetric quantum mechanics in generic non-Hermitian systems " arXiv:1906.03431 (2019).

[60] M. A. de Ponte, F. S. Luiz, O. S. Duarte, and M. H. Y. Moussa,"All-creation and allannihilation time-dependent $\mathcal{P} \mathcal{T}$-symmetric bosonic Hamiltonians: An infinite squeezing degree at a finite time" Phys. Rev. A. 100, 012128 (2019).

[61] H. R. Lewis and W. B. Riesenfeld, "An exact quantum theory of the time dependent harmonic oscillator and of a charged particle time dependent electromagnetic field," J. Math. Phys. 10, 1458 (1969).

[62] E. Wigner, Group Theory and Its Application to Quantum Mechanics of Atomic Spectra (Academic Press, New York), (1959).

[63] V. B. Berestetskii, E. M. Lifshitz and L. P. Pitaevskii, Quantum Electrodynamics (Oxford: Pergamon Press), (1982). 
[64] B. Chern and A. Tubis, "Invariance Principles in Classical and Quantum Mechanics," Am. J. Phys. 35, 254 (1967).

[65] A. de Sousa Dutra, M.B. Hott and V.G.C.S. dos Santos, "Time-dependent non-Hermitian Hamiltonians with real energies," Europhys.Lett.71, 166 (2005).

[66] C. Yuce, "Time-dependent PT-symmetric problems," Phys.Lett.A 336, 290 (2005).

[67] C. Yuce, "Complex Spectrum of a Spontaneously Unbroken PT Symmetric Hamiltonian," arXiv: 0703235v1 (2007).

[68] N. Moiseyev, "Crossing rule for a PT -symmetric two-level time-periodic system," Phys. Rev. A 83, 052125 (2011).

[69] X. Luo, J. Huang, H. Zhong, X. Qin, Q. Xie, Y.S. Kivshar, C. Lee, "Pseudo-Parity-Time Symmetry in Optical Systems," Phys. Rev. Lett. 110, 243902 (2013).

[70] Xiaobing Luo, Donglan Wu, Senping Luo, Yu Guo, Xiaoguang Yu and Qianglin Hu, "Pseudo-parity-time symmetry in periodically high-frequency driven systems: perturbative analysis," J. Phys. A 47, 345301 (2014).

[71] M. Maamache, S. Lamri and O. Cherbal, "Pseudo PT -symmetry in time periodic nonHermitian Hamiltonians systems," Annals of Physics 378, 150 (2017).

[72] The authors of Ref. [1] wrote Eq. (27), this means that eigenstates $\phi_{\lambda}(x, t)$ of the invariant operator $I(t)$ are transformed under the $\mathcal{P} \mathcal{T}$ action as $\mathcal{P} \mathcal{T} \phi_{\lambda}(x, t)=\phi_{\lambda}^{*}(-x, t)$. This implies that the eigenstates $\phi_{\lambda}(x, t)$ of $I(t)$ are NOT eigenstates of the $\mathcal{P} \mathcal{T}$ operator as claimed by the authors of Ref. [1. One can see that in the following

$$
\begin{aligned}
P T \phi_{\lambda}(x, t) & =\sqrt{\frac{\sigma_{\lambda}}{2 \pi b^{*}(t)}} \exp \left\{\frac{-i}{b^{*}(t)}\left[\left(\lambda-\gamma^{*}(t)\right)(-x)-\frac{a_{0}^{*}}{2} x^{2}\right]\right\} \\
& =\sqrt{\frac{\sigma_{\lambda}}{-2 \pi b(t)}} \exp \left\{\frac{-i}{b(t)}\left[(\lambda-c(t)) x-\frac{a_{0}}{2} x^{2}\right]\right\} \\
& \neq \phi_{\lambda}(x, t) .
\end{aligned}
$$

Morever these eigenstates of $I(t)$ will not also satisfy the normalization condition with the Dirac delta function

$$
\int_{-\infty}^{+\infty} \phi_{\lambda^{\prime}}^{P T}(x, t) \phi_{\lambda}(x, t) d x=\frac{\sqrt{\sigma_{\lambda^{\prime}} \sigma_{\lambda}}}{2 \pi}\left[\exp \frac{\left(\lambda-\lambda^{\prime}\right)}{|b(t)|} x\right]_{-\infty}^{+\infty}
$$

where $b(t)=i|b(t)|$ is purely imaginary. 
This figure "2.PNG" is available in "PNG" format from: http://arxiv.org/ps/1909.09940v1 
This figure "B.PNG" is available in "PNG" format from: http://arxiv.org/ps/1909.09940v1 
This figure "aa.PNG" is available in "PNG" format from: http://arxiv.org/ps/1909.09940v1 